\title{
Hacia una cultura de prevención de la violencia
}

\section{Towards a culture of violence prevention}

\author{
Martha Alicia Romero Echevarría* \\ Escuela Profesional de Ciencias de la Comunicación, \\ Universidad de San Martín de Porres, Perú
}

\section{Resumen}

En el mundo la violencia se ha recrudecido en todos los niveles sociales y recientemente se ha evidenciado el incremento sustancial en el problema de la violencia social y familiar. En este trabajo se presenta la percepción de la población respecto al rol que cumplen las instituciones como propulsoras de la prevención de la violencia, considerándose el Estado, la familia, la escuela, los grupos sociales y los medios de comunicación. La muestra la conforman 300 jóvenes que viven en Lima, cuyas edades oscilan entre 18 y 28 años. Mediante un cuestionario y una entrevista no estructurada las personas evaluadas señalan que se ha perdido de vista la responsabilidad de la familia como principal fuente de desarrollo personal y social, y que socialmente se han normalizado comportamientos desconectados de lo moralmente bueno, dando paso a la violencia. Por consiguiente, se plantea que los proyectos de prevención de la violencia integren un trabajo conjunto que articule las necesidades sociales con los determinantes culturales, involucrando a las personas y las instituciones.

Palabras clave: familia; violencia; cultura; prevención; educación

Este es un artículo Open Access bajo la licencia Creative 


\begin{abstract}
Violence has intensified at all social levels worldwide, generating problems of different magnitude or competence and involving people either as aggressors or victims. This paper shows the findings of the perception of the different social actors who are immersed in abuse, taking as reference the role of the State, school, family and media as the main drivers of a culture of prevention. The analysis demonstrates that people have lost sight of family responsibility as the main source of personal and social development. In addition, at the social level, behaviors disconnected from morally good actions have been normalized, giving way to violence. Therefore, this paper proposes to include violence prevention projects in a joint work that articulates social needs with cultural determinants, being necessary to establish strategies that involve social institutions and organizations.
\end{abstract}

Keywords: family; violence, culture; prevention; education

\title{
Introducción
}

Recientemente se ha evidenciado el incremento sustancial en el problema de la violencia social y familiar. Aunque las leyes son cada vez más estrictas este fenómeno no se ha logrado frenar y menos disminuir por lo que sigue evidenciándose con más agresividad y crueldad, lo que deja entrever que existe una falta de articulación entre los decisores políticos y sociales para un trabajo concertado y comprometido. La prevención, por lo tanto, debe consistir en acciones programadas, teniendo como fin modificar la conducta de los ciudadanos de manera que no incurran en actos violentos. Al respecto de estas acciones el Banco Interamericano de Desarrollo (BID) sostiene: «Tienen por objeto reducir el delito y la violencia antes de que ocurran, modificando la tendencia de los infractores a cometer actos delictivos y violentos» $(2010$, p. 7$)$.

En el Perú existen políticas de prevención de la violencia que se deben aplicar en todos los ámbitos y territorios del país. En este sentido, de acuerdo a la Ley 30364, el Observatorio nacional de la violencia contra las mujeres y los integrantes del grupo familiar le hizo seguimiento al Plan nacional contra la violencia de género programado del 2016 al 2021, cuyo objetivo es implementar 
y organizar acciones que involucren a todas las entidades del Estado en la reducción de la violencia hacia la mujer; pero los reportes anuales de los logros en el proceso no son los esperados, por cuanto se evidencia que el nivel de incidencia de violencia contra las mujeres e integrantes del grupo familiar en el 2016 fue de $10.8 \%$, en el 2017 se redujo a $10.6 \%$ y en el 2018 volvió a subir a $10.9 \%$, estas cifras evidencian que las acciones no obedecen a las propuestas ya que la expectativa es llegar a $7.7 \%$ al 2021. De la misma manera se presenta el nivel de feminicidios: en el 2016 hubo 105 casos; en el 2017 hubo 116 casos y en el 2018 la cifra fue de 150 casos. La expectativa para el 2021 es que se reduzca la cifra a 46 (Observatorio Nacional de la Violencia contra las Mujeres y los Integrantes del Grupo Familiar, 2019).

Estos resultados son desalentadores, para los ciudadanos es imprescindible contar con soluciones de corto, mediano y largo plazo. Por lo tanto, es importante conocer la percepción de la población respecto al rol que cumplen las instituciones como propulsoras de la prevención de la violencia considerándose a algunos de los principales influyentes y responsables, entre ellos, el Estado, la familia, la escuela, los grupos sociales y medios comunicación.

En principio, es necesario establecer las causas o lo que genera la violencia, ya que esta no se presenta de manera repentina ni por un evento aislado; más bien, responden a la exposición sistemática de estímulos violentos que acontecen desde edad temprana, teniendo como principales referentes a los padres. Fernández y Rodríguez (2002) lo denominan «riesgo acumulativo, el cual indica que es una conducta aprendida como estilo de vida» (p.149); es en este proceso de aprendizaje que se va normalizando y se pierde de vista el verdadero sentido de familia.

\section{Los proyectos en prevención de la violencia}

Con el tiempo se han ido implementando proyectos y programas de prevención de la violencia que responden a las necesidades sociales, personales y familiares. Por su parte, la Organización Mundial de la Salud (OMS) considera que «la violencia puede evitarse, y su prevención es una condición indispensable y fundamental para la seguridad de los seres humanos» es así que conmina a los Estados Miembros a unificar esfuerzos para trabajar en prevención de la violencia «como prioridad de salud pública» (2003, p. 2). 
Los trabajos de prevención en violencia se han venido desarrollando como iniciativas internacionales, tanto públicas como privadas, por ejemplo, en España se llevó a cabo un programa para escolares en prevención de la violencia en el noviazgo, con el objetivo de reducir el inicio de la violencia desde las primeras relaciones amorosas, los resultados mostraron que con la aplicación de «una estrategia metodológicamente adecuada para abordar esta problemática» (Muñoz, 2010, p. 62) se logró reducir la violencia en esta muestra de estudio.

Un caso cercano es el de Medellín, Colombia, con el programa de paz que se puso en marcha en tres ciudades, con acciones «en educación, recreación y resocialización para jóvenes, participación comunitaria, influencia de los medios de comunicación» (Arriagada \& Godoy, 1999, p. 42). Los resultados han sido alentadores ya que producto de la implementación de este programa ha disminuido el índice de violencia; asimismo, se está tomando como modelo en todo el territorio, respondiendo a las necesidades de cada lugar.

Las políticas de prevención son a largo plazo y en ella están comprometidos el Estado y la sociedad, se desarrollan de manera transversal e involucra a todos los ciudadanos pasando a ser una cuestión cultural. De esta forma, en una sociedad cuando ingresa una persona con costumbres diferentes debe adecuarse definitivamente, porque es una exigencia para constituirse a la sociedad, lo que pone en evidencia que hay influencia sociocultural en el aprendizaje de la violencia; de manera individual, por ser parte o estar expuesto al abuso físico y psicológico, y por cuestiones culturales.

El aprendizaje de la violencia ocurre por diferentes motivos, por ejemplo, haber sido sometido o expuesto a la violencia o por tener modelos violentos, en ambos casos se pueden producir efectos en la conducta de diferente tipo, que van desde permisividad y sometimiento hasta acciones violentas y crueldad, esto dependiendo de las características sociopersonales del sujeto. Bandura da lugar a la explicación de conductas antisociales y delictivas como aprendidas; más adelante, Akers formula un modelo que explica el aprendizaje de la conducta que se observa y modela en «la familia, grupo de pares, medios de comunicación, etc. Además, establece una serie de requisitos o factores de riesgo, desde el paradigma del aprendizaje humano que hacen más probable que una persona sea violenta» (como se citó en Montolío, Moreno, \& Robles, 2012, p. 493). 
De allí que es importante conocer el rol que cumplen cada uno de los actores sociales en la prevención de la violencia, en evaluar y reestructurar los programas para llegar a todos los niveles sociales, económicos y culturales, abriendo caminos hacia la cultura de paz.

\section{El rol del Estado en la prevención de la violencia}

El Estado peruano, hasta el momento no ha logrado desarrollar una cultura de prevención debido a que los diferentes gobiernos de turno no los culminan, a pesar de los denodados esfuerzos para generar proyectos de prevención, pues los trabajos a largo plazo o sostenibles no brindan a los gestores indicadores de logro inmediato, lo que hace que los programas terminen desapareciendo.

El Sistema nacional para la prevención, sanción y erradicación de la violencia contra las mujeres y los integrantes del grupo familiar, tiene a su cargo coordinar, planificar, organizar y ejecutar un conjunto de actividades que brinden garantía para que se cumpla con «la política pública en materia de prevención, atención, protección y reparación de la víctima, la sanción y reeducación de la persona agresora, para lograr la erradicación de la violencia contra las mujeres y los integrantes del grupo familiar» (Ministerio de la mujer y poblaciones vulnerables, 2019, párr. 1). En este contexto, se han iniciado programas de prevención establecidos del 2016 al 2021, pero que no solo no están dando resultados, sino que se ha incrementado la violencia al 2018.

Como el mencionado, hay muchos programas a nivel de gobierno local, regional y nacional que abarcan todos los sectores de Estado, que tienen como objetivo erradicar la violencia y prevenir su aparición; para lograrlo cuentan con grandes equipos, pero existe una desarticulación en el proceso de intervención que finalmente afecta la calidad de vida de los peruanos, de manera que teniendo en cuenta la diversidad cultural, es necesario contar con un enfoque integrado de gobernabilidad que permita administrar los proyectos de intervención con programas eficientes, oportunos y sostenibles.

Resolver el problema de agua, alimentación y energía es de vital importancia para la calidad de vida de la población, pero también lo es establecer una cultura de prevención de la violencia, para lograrlo se pueden 
aprovechar las herramientas que ofrece el Nexus governance ya que propone un trabajo articulado e integrado a nivel administrativo con la finalidad de lograr una adecuada gestión en torno a los recursos que maneje. Hagemann y Kirschke (2017) acotan que al gobernar «these resources in an integrated manner, problems such as resource scarcity, quality, and human wellbeing will be addressed in a more sustainable way. The concept has thus also become a cornerstone in achieving and monitoring the Sustainable Development Goals (SDGs)». [estos recursos de manera integrada, problemas como la escasez de recursos, la calidad y el bienestar humano se abordarán de una manera más sostenible. Por lo tanto, el concepto también se ha convertido en una piedra angular para lograr y monitorear los Objetivos de Desarrollo Sostenible (ODS)] (p. 1). Es importante recalcar que los últimos gobiernos no han trabajado en sostenibilidad por una concepción cortoplacista, dejando de lado las prioridades de los ciudadanos.

Es necesario advertir que quien asume un cargo en el Estado se convierte en un servidor público, y debe asumir un franco compromiso moral en bienestar de los ciudadanos, sin embargo, se ha generado desconcierto en el pueblo, por los diversos actos y sucesos en los que incurren los funcionarios y servidores públicos, que a todas luces van quedando en la impunidad, generando una normalización de la violencia estatal que generan dichos populares como «roba pero hace», «a nada», generando un divorcio entre la ética y la política. Leuridan (2016) frente a esto acota: «La vulgaridad de la corrupción a nivel de instituciones estatales y principalmente la defensa de los pobres necesitan un Estado fuerte, pero también inspirado por el bien y la justicia y no por una burocracia que bloquea la vida económica y promueve la vulgaridad» (p. 329); por lo que es necesario involucrarse en este proceso de transparencia para lograr un cambio y bienestar para todos los ciudadanos.

\section{La educación en prevención de la violencia}

En el ámbito de la educación básica recientemente se han concebido e implementado medidas que involucran a todos sus actores para prevenir y atender los casos de violencia contra los escolares, esto viene ocurriendo desde mayo del 2018, tiene como fin orientar y garantizar una buena interacción del escolar con su entorno en pro de su desarrollo integral, mediante el conocimiento de sus derechos y responsabilidades, cabe recalcar que aún no se cuenta con resultados palpables (Ministerio de Educación, 9 de mayo de 2018). 
Al parecer los padres piensan que es la escuela la que tiene que educar y fijarle reglas a sus hijos; por su parte, los profesores piensan que esta labor es responsabilidad de los padres. Al respecto Fan y Chen; Margolin y Gordis (como se citó en Fernández \& Rodríguez, 2002) afirman que «la implicación activa de los padres en la vida académica de sus hijos contribuye a mejorar el rendimiento escolar, por lo tanto, se supone, ayuda a reducir la probabilidad de emitir conductas violentas» (p. 149). En este sentido sería conveniente que se armonicen acciones en pro de actividades formativas y académicas de los estudiantes para que los ayuden a reducir comportamientos violentos.

La escuela, no cambiará la sociedad, más bien, es la sociedad la que hace a la escuela. La violencia que se genera en la escuela, en última instancia, no es más que un reflejo de la violencia socialmente establecida e históricamente legitimada (Fan \& Chen; Margolin \& Gordis, como se citó en Fernández \& Rodríguez, 2002, p. 149).

En el ámbito universitario tampoco se ha evidenciado trabajos a favor del buen vivir, buen ser o buen estar, siendo este un espacio de formación profesional. La universidad debe asumir la responsabilidad de promover el desarrollo integral de los estudiantes para cubrir las necesidades sociales y contribuir de manera sostenible al desarrollo del país desde todos los ámbitos, poniéndose al servicio de la comunidad para el desarrollo local, regional y nacional (El Peruano, 2014).

De otro lado, en la actualidad los grupos sociales promueven actividades conjuntas en las que se busca la aceptación y el aprecio entre sus pares. Pueden ser oportunidades de desarrollo del bien común y el logro social, pero también pueden constituirse en una amenaza por su capacidad para influir en la conducta de sus miembros, que se esfuerzan en demostrar lo que pueden hacer para pertenecer al grupo. En ese afán pueden caer en el mundo de las drogas o la exacerbación de la sexualidad descontrolada, convirtiéndose en personas de riesgo para ejercer o ser víctima de violencia.

Es necesario, entonces, fortalecer en el estudiante los conocimientos científicos, tecnológicos y culturales con base humanística, la capacidad para tomar decisiones con responsabilidad y sentido crítico, las actitudes para el liderazgo y desarrollo de habilidades sociales; es decir, que se encuentre 
preparado para afrontar una vida orientada hacia el bienestar personal y social, promoviendo una cultura de paz.

\section{La familia en prevención de la violencia}

Del seno familiar nacen y se forjan los ciudadanos que son quienes engrandecen la sociedad o por el contrario la pervierten, aquí el rol de los padres de familia es determinante y valioso. Cuando la familia entra en crisis se «afecta la parte más importante del ser humano, la educación en los valores que se recibe principalmente en la familia» (Leuridan, 2016, p. 46); ocurre la construcción o destrucción de sus miembros.

En el cuarto artículo del capítulo II de la Constitución Política del Perú (1993) se detalla la protección a la familia y la promoción del matrimonio: $L a$ comunidad y el Estado protegen especialmente al niño, al adolescente, a la madre y al anciano en situación de abandono. También protegen a la familia y promueven el matrimonio. Reconocen a estos últimos como institutos naturales y fundamentales de la sociedad.

Los roles en las familias han sufrido cambios, con el transcurrir del tiempo tomaron nuevos rumbos, las mujeres se fueron posicionando en los ámbitos político, social y económico que habían sido ocupados casi exclusivamente por los hombres. También, se transformó la concepción del «hombre de la casa» -la persona encargada de proteger, cubrir necesidades económicas, afectivas y sociales de su familia- en una de pertenencia, machismo sutil o recalcitrante que lo condujo a la creencia de «la supremacía del hombre y en la inferioridad de la mujer» (Ferrer \& Bosh, 2000, p. 16). En este ejercicio de poder predomina la falta de tolerancia y control emocional, desencadenando frecuentemente en actos violentos contra la propia familia y en particular contra la pareja; teniendo como consecuencia la destrucción o descomposición de la familia, la pérdida de los valores personales y sociales, cayendo en lo que se denomina reducción del signo a apariencia, por cuanto la familia siendo el eje de toda sociedad y propulsora de valores cae en «las ideologías dominantes, procedentes de una mentalidad común», por lo que muchas familias hacen silencio y soportan la violencia manteniendo esta condición en privado, hasta que se vuelve insostenible por sus consecuencias, muchas veces fatales (Guissani, 2003, p. 107). 
El inicio del ejercicio de la violencia es engañoso, en la mayoría de los casos se introduce sin avizorar su aparición y posicionamiento. En este proceso, de manera progresiva, el agresor aísla a la víctima de familiares o personas que la pueden proteger, desmerece sus cualidades personales, físicas, intelectuales y sexuales, mellando su dignidad y valía personal, generando dependencia emocional, sentimiento de pertenencia y poca valía personal; todo esto, empodera al agresor, aumenta su ego y al sentirse superior ejerce el dominio develando la conducta violenta que cada vez es más intensa y frecuente. Mientras que la persona violenta tiene sentimientos de complacencia y placer, los demás miembros de la familia soportan el dolor ocasionado por la violencia emocional, física, sexual y/o económica.

Producto de lo mencionado, se produce un desequilibrio emocional por una dicotomía afectiva provocada por el sentimiento de amor y odio hacia la persona que protege, pero que al mismo tiempo causa daño y violenta. Por ejemplo, hace unos años vino a consulta un joven estudiante de derecho y pedía apoyo para su madre, ambos habían sido golpeados sistemáticamente por el padre y nunca habían denunciado este hecho; esgrimía como razón: es mi papá, yo nunca le haría eso. Unos años después regresó a consulta, este joven había perdido el control con su pareja y la había golpeado. Esto es una clara muestra de cómo influye la familia en la producción de víctimas y victimarios con problemas internos que luego se convierten en problemas sociales. Al respecto Leuridan (2016) cuestiona: «cómo podemos aprender a manejar el conflicto ambivalente en nosotros mismos, o sea el hecho de que queramos eliminar y destruir a las mismas personas que son importantes para nosotros y a las que queremos de todo corazón» (p. 319).

Las consecuencias de la violencia intrafamiliar se extienden por generaciones y se agudizan con el paso del tiempo, debido a la aparición de nuevas formas de violentar y de violentarse, vale decir que los hijos de la violencia no siempre son resilientes, por lo tanto, en la búsqueda de su equilibrio y de minimizar el dolor emocional y vacíos existenciales son propensos a buscar refugio y caer en distintas formas de violencia que van desde el bajo rendimiento académico, deserción educativa, consumo de drogas, pandillaje, relaciones sexuales dispersas, etc.; dichas inconductas a su vez provocan el maltrato de sus padres y con esto se justifican. Se vuelve un espiral de violencia. 


\section{Los medios de comunicación en prevención de la violencia}

Temas que se han exacerbado por los medios de comunicación son los de contenido violento, especialmente familiar y de género, sin caer en cuenta en lo contraproducente que pueden ser al influir en la audiencia. García (2007) señala que «los contenidos y las formas narrativas se seleccionan teniendo como criterio supremo el impacto que puedan causar en la audiencia, en lugar de la capacidad de suministrar información relevante, de la forma más rigurosa posible» (p. 51).

Los medios masivos (periódicos, televisión, radio, Internet) propagan las noticias de distintas formas; no solo muestran hechos reales, actuales y de interés social, sino también hechos poco confiables o veraces. Para Redondo (2011) es importante brindarle a los ciudadanos «noticias esenciales que precisan para ejercer sus derechos cívicos-dado que en los medios dominan las informaciones más ligeras o triviales-, la democracia que reside en la voluntad popular acabará por banalizarse o resultar altamente manipulable» (p. 10).

Se puede afirmar que las personas son individuos psicológicamente activos, la pertenencia a grupos sociales de ninguna manera es indicador de una reacción homogénea, es así que «los individuos que son expuestos a información nueva o que no va de acuerdo a sus valores experimentan algo llamado disonancia cognoscitiva» (Carl Hovland como se citó en Fernández \& Galguera, 2009, p. 102). Como ejemplo, se menciona la producción de Orson Welles, cuando transmitió por radio La Guerra de los Mundos asegurando que la tierra sería atacada por extraterrestres; las reacciones fueron diversas, muchos tuvieron miedo y salieron de sus casas, pero otros simplemente no; lo que lleva a la pregunta ¿por qué algunos fueron invadidos por el pánico y otros simplemente no lo creyeron? Klapper hizo referencia al poder limitado de los medios, acotando: «Lo que llevó a pensar que más que cambiar a las personas, los medios refuerzan actitudes ya existentes» (como se citó en Fernández \& Galguera, 2009, p. 103).

La forma de presentar las noticias respecto a la violencia familiar y de género recaen en el sensacionalismo como un proceso sistemático que influye en la audiencia de manera subjetiva. Con el objetivo de capturar la atención, se sobredimensiona los aspectos negativos del suceso. Mejía (2004) sostiene: 
«Su intensión primordial es provocar la noticia si ésta no existe o, en cualquier caso, deformarla» (p. 11).

Por lo tanto, y de acuerdo a lo observado, los fines supremos de la información periodística no se cumplen; hace falta formar una cultura de prevención para promover la toma de conciencia social y dilucidar soluciones orientadas a tomar decisiones saludables, lo que sería posible de existir un trabajo comprometido. Burns, afirma: «Los medios masivos pueden alcanzar objetivos sobre otras personas a voluntad de quienes los dirigen, poseen o controlan, y quienes se valen de ellos para canalizar sus mensajes» (como se citó en Castillo, 2011, p. 5).

Por consiguiente, el objetivo del presente trabajo es dilucidar la responsabilidad que tienen los actores sociales en la promoción o prevención de la violencia, el rol que cumple el Estado, la familia, la educación y los medios de comunicación, con el fin de promover una cultura de prevención de la violencia personal, familiar y social.

\section{Método}

\section{Diseño}

La presente investigación es de diseño no experimental, de enfoque mixto, y nivel descriptivo-explicativo.

\section{Participantes}

La muestra, elegida al azar, la integran 300 jóvenes que viven en Lima, cuyas edades oscilan entre 18 y 28 años; siendo $52 \%$ mujeres y $48 \%$ varones, de diferentes niveles educativos; $72 \%$ con estudios universitarios. Se utilizó un muestreo probabilístico para seleccionar la muestra.

\section{Instrumentos}

- Cuestionario: Prevención de la violencia (Anexo 1). Consta de 6 ítems centrales; los tres primeros tienen subítems que en total suman 25; dos ítems son de respuesta cerrada y uno de respuesta abierta. Las 
alternativas de respuestas tienen una escala del 1 al 5, donde 1 es el menor nivel y 5 el mayor nivel.

- Entrevista no estructurada a 20 jóvenes, quienes brindaron su opinión acerca del rol de los padres en la violencia familiar.

\section{Procedimiento}

Se contó con la autorización respectiva para recabar la información en seis aulas de la universidad nacional y dos de la universidad particular. Se obtuvo 314 cuestionarios, de los cuales se excluyeron 14 por falta de alguna información.

El procesamiento de los datos cuantitativos ha servido para complementar el análisis cualitativo en una triangulación de las entrevistas, las preguntas abiertas, la observación, la experiencia de la investigadora que se ha recogido en los años de trabajo en prevención de la violencia social y familiar y en las investigaciones científicas que respaldan el estudio.

\section{Resultados}

El análisis cualitativo de las entrevistas realizadas a los estudiantes se contrasta con las respuestas obtenidas a nivel cuantitativo que muestran resultados porcentuales de acuerdo a cada ítem o pregunta formulada en el cuestionario Prevención de la violencia, lo que a su vez, ha permitido establecer las siguientes interpretaciones (ver Anexo 2):

- La mayoría de jóvenes perciben que el Estado es el segundo responsable en generar una cultura de prevención de la violencia con objetivos claros y promover el respeto de las leyes desde sus propios espacios, ya que no hay una debida fiscalización. El 39\% le otorgó la mayor responsabilidad al Poder Judicial, $34 \%$ al Poder Legislativo y 27\% al Poder Ejecutivo.

- En las respuestas emitidas acerca del rol de la familia en la prevención de la violencia, indican a la familia como gestor principal, porque modela las actitudes, valores, creencias, autoestima y comportamientos sociales de los menores. Resulta alarmante que el $42.7 \%$ de los encuestados reconoce que 
alguna vez fueron víctimas de violencia familiar, además, señalando responsable al padre $51 \%$ y $49 \%$ a la madre ya sea porque permite la violencia o por que la ejerce. El $40 \%$ opina que la persona soporta violencia porque ha sido maltratada en su infancia; $31 \%$ porque hay diferencias en el nivel de educación; y $29 \%$ porque ganan menos dinero.

- Los entrevistados consideran que los centros educativos deben ser apoyo para formar de manera integral futuras generaciones, y los profesores deben ser ejemplo de conocimientos y actitudes para los alumnos, teniendo una tarea importante en la prevención de la violencia; sin embargo, en muchas oportunidades ocurre lo contrario, como cuando algún profesor se burla de un estudiante, o cuando permite que los otros se burlen, o cuando les dejan mucha tarea para la casa, provocando molestia en los padres que la descargan en sus hijos. De acuerdo a estas respuestas, el $44 \%$ de los encuestados otorga responsabilidad a los centros educativos; el 30\% a los institutos y el 29\% a las universidades. Los centros educativos son responsables de asumir y ejecutar proyectos de prevención sostenible desde edades tempranas.

- Los medios de comunicación deben servir como el principal propulsor de una cultura de paz, no obstante, presentan contenidos violentos dándole énfasis al morbo especialmente cuando se trata de feminicidios, robos, asaltos y muerte violenta. Mediante los resultados observamos que el 29\% opina que en la televisión se muestra mayor cantidad de contenido violento, el $24 \%$ opina que en las redes, el $18 \%$ en las películas, el $17 \%$ en las letras de las músicas y el $12 \%$ en el streaming.

\section{Discusión}

Si bien es cierto que se le otorga al Estado una responsabilidad inherente a su función de velar por la seguridad y bienestar social, los proyectos de prevención de la violencia deben darse de manera sostenible, dejando de lado los intereses reeleccionistas que no permiten que se den a largo plazo y menos aún que sean sostenibles. El estudio nos permite aseverar que la percepción de los ciudadanos acerca de los sistemas de gobierno y de sus integrantes es negativa, a esto se suman los resultados obtenidos desde el Observatorio nacional de violencia contra las mujeres y los integrantes del grupo familiar, que 
implementó el plan nacional contra la violencia de género para el 2016-2021, logrando reducirla de $11.7 \%$ a 10.8\% para el 2016; al 2017 disminuyó a 10.6\% y para el 2018 volvió a aumentar a 10.9\%, lo cual indica que no se han tomado en cuenta todos los factores de la violencia psicológica, física y sexual contra la mujer, situación que demuestra que las acciones no se han articulado de manera eficaz, siendo necesario realizar el análisis de todos sus elementos y cambios estructurales para mejorar las políticas públicas en la prevención de la violencia.

Personas de diferentes contextos sociales: universitarios de pregrado y posgrado; empresarios, mandos medios, sindicalistas; y trabajadores d las cortes superiores en las visitas judiciales, respondieron acerca de la percepción de los ciudadanos sobre los presidentes, congresistas y jueces; señalaron que lo que tienen en común es que son corruptos y que mienten. Resultando ser un claro indicador que tanto las personas como los gestores han normalizado la violencia, los medios evidencian actos de corrupción y violencia en los poderes del Estado y «no pasa nada», cayendo en un reduccionismo, de manera que son modelos «que influyen en los comportamientos. Para hablar de moral entonces, es importantísimo que comprendamos y tomemos conciencia del tipo de cultura a la que pertenecemos» (Giussani, 2003, p. 104).

Con el devenir del tiempo las familias han asumido diferentes conformaciones y roles sociales, pero definitivamente, siguen siendo los padres los que marcan la vida de sus hijos ya que de ellos asumen una cultura. «La familia es el lugar de la formación integral, donde se desenvuelven los distintos aspectos, íntimamente relacionados entre sí, de la maduración personal» (papa Francisco, 2015).

Por medio de los resultados se evidencia que la mayoría de jóvenes consideran que es importante contar con una familia, ya que mediante ella se moldea la personalidad, siendo los padres quienes deben educar con valores y disciplina a los hijos, pero también, la familia puede convertirse en el principal promotor de la violencia que afecta física y psicológicamente a los niños. En este sentido, Hinojosa y Vásquez (2018) aseveran que es necesario prestar atención a «las relaciones familiares y a su influencia en el desarrollo del comportamiento del individuo en tiempo presente y futuro» (p. 435) los niños violentados se convierten en adolescentes y luego ciudadanos violentos, con poca capacidad para establecer empatía, marchando hacia la búsqueda 
de una incesante autocomplacencia. Le otorgan estatus a la violencia personal y social en la cual queda sumida toda la familia.

Los centros educativos y de formación tienen un rol social frente a la prevención de la violencia, en mayo del 2018 Daniel Alfaro, el entonces Ministro de Educación, anunció nuevas medidas para frenar la violencia en los colegios, estas incluían la incorporación de psicólogos, asistentes sociales y otros profesionales, el objetivo fue atender y prevenir casos de violencia entre los estudiantes y hacia ellos. Uno días después anunció el proceso de contratación de los profesionales y capacitación a más de 13 mil directores para mejorar sus habilidades en el manejo de la convivencia escolar (MINEDU, 15 de mayo de 2018). La ciudadanía aún deberá esperan los resultados, pues hasta el momento no se han evidenciado cambios positivos.

La presente investigación nos conduce a reconocer que en los centros educativos se presentan todo tipo de violencia y muchas veces los profesores prefieren no involucrarse debido a la falta de protección que perciben tener. En cuanto a los alumnos que vienen de sus hogares con huellas de maltrato las acciones son limitadas. En la Resolución Ministerial emitida en setiembre del 2007, se aprobaron los lineamientos de acción en caso de maltrato a estudiantes en los centros educativos, una medida necesaria por la coyuntura del momento; sin embargo, producto de la interpretación del artículo $1^{\circ}$ donde se resuelve aprobar «Lineamientos de acción en caso de maltrato físico y/o psicológico», se limitan las acciones formativas y disciplinarias en las aulas de los centros educativos, pues empiezan a conocerse denuncias afectuadas a los profesores por parte de los escolares y sus familiares al sentir que son maltratados psicológicamente.

Si las universidades forman los profesionales que las sociedades requieren, entonces lo que se necesita en la actualidad es salir del caos social, producto de la exacerbación de la violencia en la sociedad peruana, para ello, es necesario «formar profesionales de alta calidad de manera integral y con pleno sentido de responsabilidad social de acuerdo a las necesidades del país» (Ley Universitaria, artículo 6.2). Por lo tanto, todos los profesionales deben estar preparados para asumir una cultura de prevención de la violencia, promoviendo y poniendo en marcha proyectos sostenibles, eficientes y eficaces que cuenten con su permanentemente monitoreo. 
Los medios de comunicación se convirtieron en una fuente de información y ahora de interacción permanente con las personas, están cargados de contenido de todo tipo, y varias teorías y estudios diversos ponen en evidencia el poder que tienen en la percepción de los usuarios. Los contenidos violentos se presentan de diferentes formas y para todas las preferencias ya que van en juegos interactivos, dibujos animados, novelas, programas informativos, series, redes sociales, etc.; dichos contenidos acercan a las personas a la destrucción y muerte, al feminicidios y violencia social con el único fin de ganar audiencia, Castrillo (2014) señala que los medios prefieren «transferir mensajes banales, exagerados e indecentes, con el propósito de crear sensaciones que ocasionen cambios en el estado de ánimo de la audiencia, que permita acrecentar sus rendimientos económicos producto de la labor comercial» (p. 4). Con relación a estos contenidos, estudiantes del último año de periodismo opinaron que la preparación que reciben en su formación profesional es buena, pero que al enfrentarse al campo laboral -teniendo en cuenta la ética-deben adaptarse, de lo contrario no sobrevivirían en los medios.

En acciones propuestas para la prevención de la violencia, todos los medios participaron el 2015 en la marcha Ni una menos, a pesar de ello, lo que se ha logrado es exacerbarla. Otro ejemplo, el 2017 por el Día Internacional de la Mujer, en televisión se presentó la campaña publicitaria \#noesnormal, que «muestra la realidad de la violencia que muchas personas esconden por motivos personales o sociales, orientándolas hacia la reflexión, estimulando al cambio, mostrando que existe una vida diferente» (Chávarry, 2018, p. 13), sin embargo, solo estuvo disponible las primeras horas de ese día y la retiraron, luego no se hizo ningún comentario al respecto.

Los medios están dando un giro a la información vertida, en la actualidad los usuarios son más activos y buscan las formas de acercarse a la información directa y la contrastan, ejerciendo su derecho a estar informado con la verdad. Esta participación abre las puertas al ejercicio libre, crítico y ético en los medios «con un escenario mediático mucho más diverso e incontrolable, donde las fronteras (tecnológicas, jurídicas, geográficas) tienden a difuminarse» (Sampedro, 2014, p. 233). Entonces, se podrá trabajar libremente hacia una cultura de prevención de la violencia para lograr reestablecer las fuentes de paz. 


\section{Conclusiones}

Es necesario articular acciones conjuntas en todos los niveles del Estado, de manera que prevalezca una cultura de prevención de la violencia familiar y social. Las gestiones de los trabajadores, funcionarios y servidores públicos deben ser de acceso publico, de manera que la ciudadanía recobre la confianza en el abordaje de los programas y proyectos en pro de la erradicación de la violencia.

El rol de los padres es fundamental en la formación de los hijos por ser el modelos a seguir. En esta investigación se ha develado que la mayoría de los jóvenes perciben que sus padres están ocupados en sus proyectos y que ponen por encima sus necesidades individuales; de allí que es necesario trabajar los factores resilientes de los miembros de la familia desde sus espacios de desarrollo, con el fin de retomar el diálogo entre padres e hijos y recobrar el sentido de la familia como el principal factor protector de la violencia.

Se debe proponer programas de prevención de la violencia dirigido a los padres y madres de familia que trabajan en diferentes organizaciones, empresas e instituciones públicas y privadas. Asimismo, programar talleres para el desarrollo de habilidades blandas, inteligencia emocional y resiliencia con el objetivo de promover una mejor calidad de vida e integración familiar.

La educación tiene un rol preponderante frente a la prevención de la violencia, enseñando y difundiendo los derechos y deberes y obligaciones de los estudiantes, profesores y todos los miembros de la comunidad educativa. El desarrollo de las habilidades intelectuales debe estar a la par del desarrollo de los valores humanos como la solidaridad, equidad, justicia y respeto a la diversidad, de manera que el estudiante sea capaz de tomar decisiones con sentido crítico y alineado a una formación integral que lo prepare para vivir y convivir en pro de la sociedad. 


\section{Referencias}

Arriagada, I., \& Godoy, L. (1999). Seguridad ciudadana y violencia en América Latina: diagnóstico y políticas en Ios años noventa. Santiago de Chile: CEPAL. Recuperado de https://repositorio.cepal.org/handle/11362/ 6263

Banco Interamericano de Desarrollo - BID. (2010). Prevención del delito y la violencia en América Latina y el Caribe: Evidencias de las intervenciones BID. Washington, D.C.: Oficina de evaluación y desarrollo, OVE.

Castillo, A. (2011). Los medios de comunicación como actores sociales y políticos. Poder, Medios de Comunicación y Sociedad. Razón y Palabra, (75), 5-21.

Castrillo, C. (2014). El sensacionalismo. Algunos elementos para su comprensión y análisis. España: Alianza Editorial.

Chávarry, F. M. (2018). Relación del discurso publicitario y la publicidad social \#NOESNORMAL Saga Falabella en mujeres de la Molina, 2017 (Tesis de maestría). Universidad de San Martín de Porres, Lima, Perú.

El Peruano (9 de julio de 2014). Ley № 30220, Ley Universitaria. Recuperado de https://www.sunedu.gob.pe/ wp-content/uploads/2017/04/Ley-universitaria-30220.pdf

Fernández, C., \& Galguera, L. (2009). Teorías de la comunicación. Ciudad de México: Editorial Mc Graw Hill.

Fernández, L., \& Rodríguez, F. J. (2002). Prevención de la violencia: hechos y mitos. Psicothema, 14 (Supl.).

Ferrer, V. A., \& Bosch, E. (2000). Violencia de género y misoginia: reflexiones psicosociales sobre un posible factor explicativo. Papeles del Psicólogo, (75), 13-19. Recuperado de https://www.redalyc.org/pdf/778/ 77807503.pdf

García, J. A. (2007). El infoentretenimiento en los informativos líderes de audiencia en la Unión Europea. Anàlisi, 35, 47-63. Recuperado de https://core.ac.uk/download/pdf/13271691.pdf

Giussani, L. (2003). El hombre y su destino. En camino. Madrid: Ediciones Encuentro.

Hagemann, N., \& Kirschke, S. (2017). Key Issues of Interdisciplinary NEXUS Governance Analyses: Lessons Learned from Research on Integrated Water Resources Management. Resources, 6(9), 130-138. doi: 10.3390/resources6010009

Hinojosa, M. B., \& Vásquez, R. L. (2018). La familia como elemento mediador entre la cultura de paz y la violencia cultural. Justicia, 23(34), 405-455.

Leuridan, J. (2016). El sentido de las dimensiones éticas de la vida (1ª edición). Lima: Fondo editorial USMP.

Mejía, C. (2004). Cultura popular en el diseño de la prensa sensacionalista limeña. Análisis de las portadas de Ajá, Trome y el popular (abril-junio 2004) (Tesis de Licenciatura). Universidad de San Martín de Porres, Lima, Perú.

Ministerio de Educación - MINEDU. (09 de mayo de 2018). Ministro de Educación anuncia nuevas medidas para frenar la violencia en colegios públicos y privados. Recuperado de http://www.minedu.gob.pe/n/ noticia.php?id=46622

Ministerio de Educación - MINEDU. (15 de mayo de 2018). Minedu y MIMP refuerzan la convivencia escolar para prevenir la violencia en colegios públicos y privados. Recuperado de: http://www.minedu.gob.pe/n/ noticia.php?id=46698

Ministerio de la mujer y poblaciones vulnerables. (2019). Sistema Nacional para la prevención, sanción y erradicación de la violencia contra las mujeres e integrantes del grupo familiar. Recuperado de https:/l observatorioviolencia.pe/sistema-nacional/que-es/ 
Montolío, C. A., Moreno, M. C. B., \& Robles, J. L. A. (2012). La teoría del aprendizaje social como modelo explicativo de la violencia filio-parental/The social learning theory as explicative model of child-parent violence. Revista Complutense De Educación, 23(2), 487-511. Recuperado de https://search.proquest.com/ docview/1432986861?accountid=14747

Muñoz, M. J. (2010). Validación de un programa de prevención de la violencia en las relaciones de noviazgo de jóvenes y adolescentes. Madrid: Instituto de la Mujer.

Observatorio Nacional de la Violencia contra las Mujeres y los Integrantes del Grupo Familiar. (2019). Recuperado de https://observatorioviolencia.pe/el-observatorio/el-observatorio/

Organización Mundial de la Salud - OMS. (2003). Aplicación de las recomendaciones del Informe mundial sobre la violencia y la salud: informe de la Secretaría (NEB111/11). Organización Mundial de la Salud.

Papa Francisco (2015). Carta Encíclica Laudato Sí: sobre el cuidado de la casa común. Madrid: Ediciones Palabra S. A.

Redondo, M. (2011). El sensacionalismo y su penetración en el Sistema mediático español (Tesis doctoral). Universidad de Valladolid, España. Recuperado de file:///C:/Users/MariSandy16/Downloads/TESIS139120206-R.pdf

Sampedro, V. (2015). El cuarto poder en red. Por un periodismo (de código) libre. Dígitos, 1, 232-234. Recuperado de https://core.ac.uk/download/pdf/71058593.pdf 


\section{ANEXO 1 \\ CUESTIONARIO: PREVENCIÓN DE LA VIOLENCIA}

La encuesta es anónima, por eso le pedimos que respondas con sinceridad las siguientes aseveraciones desde su percepción, en una escala del 1 al 5, donde 1 es en menor grado o de nivel menor y 5 es de mayor grado o nivel mayor

1. ¿En qué medida son RESPONSABLES de la generación de violencia familiar? El Estado

$\begin{array}{lllllll}1 & \text { El Poder Judicial } & 1 & 2 & 3 & 4 & 5 \\ 2 & \text { El Poder Ejecutivo } & 1 & 2 & 3 & 4 & 5 \\ 3 & \text { El Poder Legislativo (Congreso) } & 1 & 2 & 3 & 4 & 5\end{array}$

La familia:

$\begin{array}{lllllll}4 & \text { Familia de origen } & 1 & 2 & 3 & 4 & 5 \\ 5 & \text { Papá (o quien cumple su rol) } & 1 & 2 & 3 & 4 & 5 \\ 6 & \text { Mamá (o quien cumple su rol) } & 1 & 2 & 3 & 4 & 5\end{array}$

Los centros educativos:

$\begin{array}{lllllll}7 & \text { Escuela } & 1 & 2 & 3 & 4 & 5 \\ 8 & \text { Institutos } & 1 & 2 & 3 & 4 & 5 \\ 9 & \text { Universidades } & 1 & 2 & 3 & 4 & 5\end{array}$

Las comunicaciones:

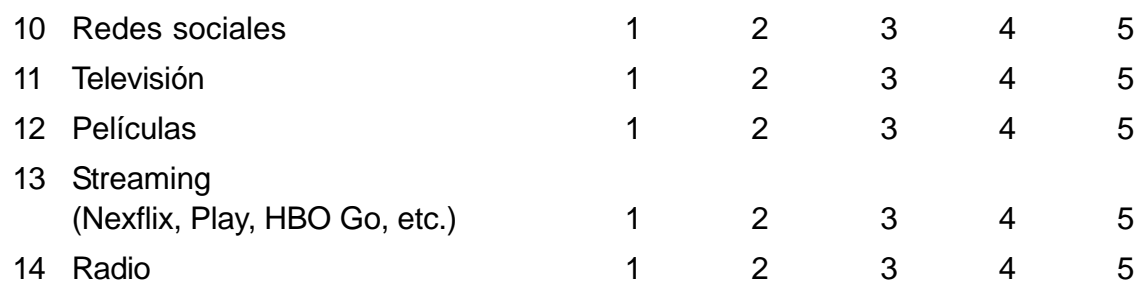

Consumo de:

$\begin{array}{lllllll}15 & \text { Alcohol } & 1 & 2 & 3 & 4 & 5 \\ 16 & \text { Drogas } & 1 & 2 & 3 & 4 & 5\end{array}$


2. ¿En qué medida las siguientes situaciones representan los motivos por los que una persona debe soportar la violencia?

20 Porque ha sido maltratada en su infancia

$\begin{array}{lllll}1 & 2 & 3 & 4 & 5\end{array}$

21 Porque hay diferencias en el nivel educación

$12 \quad 2 \quad 3 \quad 4 \quad 5$

22 Porque el Estado no le protege

$12 \quad 2 \quad 3 \quad 4 \quad 5$

3. ¿En qué medida las siguientes situaciones representan los motivos porque las personas agreden?

23 Porque ha sido maltratada en su infancia

24 Porque hay diferencias en el nivel educación

25 Porque el Estado no le castiga

$\begin{array}{lllll}1 & 2 & 3 & 4 & 5 \\ 1 & 2 & 3 & 4 & 5 \\ 1 & 2 & 3 & 4 & 5\end{array}$

4. Responda sinceramente si alguna vez ha sido parte de la violencia familiar

Sí NO

5. ¿A quién le corresponde prevenir que las personas incurran en violencia?

El Estado La familia La escuela Los medios

6. ¿Cuál sería la mejor forma de prevenir que ocurra la violencia familiar? 


\section{ANEXO 2}

\section{RESULTADOS DEL CUESTIONARIO}

\section{Resultados descriptivos del cuestionario en prevención de la violencia}

1. ¿En qué medida son RESPONSABLES de la generación de violencia familiar?

$\begin{array}{lcccccc}\text { El Estado: } & \mathbf{1} & \mathbf{2} & \mathbf{3} & \mathbf{4} & \mathbf{5} & \text { Total } \\ 1 \text { El Poder Judicial } & 3.9 \% & 9.8 \% & 19.6 \% & 29.4 \% & 37.3 \% & 100 \% \\ 2 \text { El Poder Ejecutivo } & 4.9 \% & 15.7 \% & 33.3 \% & 34.3 \% & 11.8 \% & 100 \% \\ 3 \text { El Poder Legislativo (Congreso) } & 4.9 \% & 7.8 \% & 19.6 \% & 39.2 \% & 28.4 \% & 100 \% \\ \text { La familia: } & \mathbf{1} & \mathbf{2} & \mathbf{3} & \mathbf{4} & \mathbf{5} & \text { Total } \\ 4 \text { Familia de origen } & 0 \% & 3.9 \% & 12.7 \% & 38.2 \% & 45.1 \% & 100 \% \\ 5 \text { Papá (o quien cumple su rol) } & 1 \% & 1 \% & 16.7 \% & 38.2 \% & 43.1 \% & 100 \% \\ 6 \text { Mamá (o quien cumple su rol) } & 1 \% & 1 \% & 17.6 \% & 38.2 \% & 42.3 \% & 100 \% \\ \text { Los centros educativos: } & \mathbf{1} & \mathbf{2} & \mathbf{3} & \mathbf{4} & \mathbf{5} & \text { Total } \\ 7 \text { Escuela } & 2.2 \% & 2.9 \% & 34.3 \% & 35.3 \% & 25.5 \% & 100 \% \\ 8 \text { Institutos } & 2.9 \% & 20.6 \% & 33.3 \% & 23.5 \% & 19.6 \% & 100 \% \\ 9 \text { Universidades } & 3.9 \% & 20.6 \% & 32.4 \% & 21.6 \% & 21.6 \% & 100 \% \\ \text { Las comunicaciones: } & \mathbf{1} & \mathbf{2} & \mathbf{3} & \mathbf{4} & \mathbf{5} & \text { Total } \\ 10 \text { Redes sociales } & 2 \% & 4.9 \% & 22.5 \% & 44.1 \% & 26.5 \% & 100 \% \\ 11 \text { Televisión } & 2 \% & 6.9 \% & 21.6 \% & 35.3 \% & 34.3 \% & 100 \% \\ \text { 12 Películas } & 3.9 \% & 19.6 \% & 30.4 \% & 23.5 \% & 22.5 \% & 100 \% \\ \text { 13 Streaming } & & & & & & \\ \quad \text { (Nexflix, Play, HBO Go, etc.) } & 4.9 \% & 22.5 \% & 27.5 \% & 31.4 & 13.7 \% & 100 \% \\ \text { 14 Radio } & 5.9 \% & 18.6 \% & 21.6 \% & 26.5 \% & 27.5 \% & 100 \% \\ \text { Consumo de: } & \mathbf{1} & \mathbf{2} & \mathbf{3} & \mathbf{4} & \mathbf{5} & \text { Total } \\ \text { 15 Alcohol } & 4.9 \% & 4.9 \% & 6.7 \% & 31.4 \% & 52 \% & 100 \% \\ \text { 16 Drogas } & 5.9 \% & 2.9 \% & 5.9 \% & 22.5 \% & 62.7 \% & 100 \%\end{array}$


2. ¿En qué medida las siguientes situaciones representan los motivos por los que una persona debe soportar la violencia?

$\begin{array}{lcccccc}\begin{array}{l}\text { Por qué soportar la violencia } \\ \text { 20 Porque ha sido maltratada } \\ \quad \text { en su infancia }\end{array} & \mathbf{1} & \mathbf{2} & \mathbf{3} & \mathbf{4} & \mathbf{5} & \text { Total } \\ \begin{array}{l}\text { 21 Porque hay diferencias en } \\ \quad \text { el nivel educación }\end{array} & 5.9 \% & 9.9 \% & 21.6 \% & 45.1 \% & 29.4 \% & 100 \% \\ \begin{array}{l}\text { 22 Porque gana menos dinero } \\ \text { P }\end{array} & 4.8 \% & 16.7 \% & 24.5 \% & 37.3 \% & 16.7 \% & 100 \%\end{array}$

3. ¿En qué medida las siguientes situaciones representan los motivos porque las personas agreden?

$\begin{array}{lcccccc}\text { Por qué ejercer la violencia } & \mathbf{1} & \mathbf{2} & \mathbf{3} & \mathbf{4} & \mathbf{5} & \text { Total } \\ \begin{array}{c}23 \text { Porque ha sido maltratada } \\ \quad \text { en su infancia }\end{array} & 1 \% & 2.9 \% & 16.7 \% & 48 \% & 31.4 \% & 100 \% \\ \begin{array}{c}24 \text { Porque hay diferencias en } \\ \quad \text { el nivel educación }\end{array} & 9.8 \% & 14.7 \% & 23.5 \% & 30.4 \% & 21.6 \% & 100 \% \\ \begin{array}{l}25 \text { Porque gana más } \\ \quad \text { cantidad de dinero }\end{array} & 5.9 \% & 14.7 \% & 29.4 \% & 37.3 \% & 12.7 \% & 100 \%\end{array}$

4. Responda sinceramente si alguna vez ha sido parte de la violencia familiar

$\begin{array}{ccc}\text { sí } & \text { NO } & \text { Total } \\ 42.7 \% & 57.3 \% & 100 \%\end{array}$

5. ¿A quién le corresponde prevenir que las personas incurran en violencia?

$\begin{array}{ccccc}\text { El Estado } & \text { La familia } & \text { La escuela } & \text { Los medios } & \text { Total } \\ 34.2 \% & 62.2 \% & 2.4 \% & 1.2 \% & 100 \%\end{array}$

6. ¿Cuál sería la mejor forma de prevenir que ocurra la violencia familiar? 